Editorial

\title{
Crossroads of primary and secondary cervical cancer prevention strategies in resource- constrained settings
}

\section{Editorial}

Cervical cancer represents a rare long-term consequence of persistent high-risk HPV infections of the lower genital tract, especially in settings with high HPV prevalence and poor or absent cervical screening infrastructure. Besides racial and ethnic disparities, several other factors can be implicated in the observed plateau in the incidence, mortality, and survival of cervical cancer incidence worldwide. ${ }^{1}$ In particular, poor access to health care arising by social and cultural barriers leads to inconsistent management of early cervical abnormalities, while non-attendance and high default rates from cervical screening are also common. ${ }^{2}$ Lack of physician awareness or patient's ignorance of their personal risk for cervical cancer and fatalistic attitudes toward cancer discourage participation in cervical screening. Furthermore, financial barriers in uninsured or underinsured patients, fear of pain and discomfort from a gynecologic examination and language obstacles in multiethnic populations to decrease participation in secondary cervical cancer prevention. Besides, overtreatment of patients when clinicians fear future noncompliance leads to heightened patient anxiety and community mistrust of the health care system. ${ }^{1}$ Nonetheless, lower socioeconomic status and inequalities represent fundamental problems which must be addressed in the new integrated strategies. ${ }^{3}$

More than 10years have elapsed since the global launch of the first vaccine targeting an STI, namely the quadrivalent VLP HPV vaccine. Despite being highly successful, HPV vaccination programs have been particularly well executed in special settings where their implementation has been school-based. ${ }^{4}$ The patchy coverage of vaccination target groups and the huge debate over HPV vaccination essentially stemmed from physicians and people's mixed attitudes towards vaccination, inaccurate information in the electronic and social media and the robust anti-vaccination movement among others. In hindsight, anecdotally some would argue that if the quadrivalent vaccine was globally marketed as an anti-wart vaccine without reference to cervical "precancer", it might have enjoyed more success.

In the meanwhile, the inherent drawbacks of cytology (subjective nature, low sensitivity for detecting true cervical precancer requiring frequent re-screening) have been partially addressed with the wider implementation of HPV-related biomarkers which represent objective and more repetitive methods to screen for precancerous lesions. ${ }^{5}$ Several DNA and mRNA HPV assays have gained FDA approval, other biomarkers (p16/Ki67 dual stain, viral genotyping, methylation markers) are being successfully used in daily clinical practice, and several others are still being validated in the assessment of HPV positive women. ${ }^{6}$ This progress in HPV science has been the basis of calibrated cervical screening and triage options, and guidelines endorsed by the scientific committees.

Therefore, the two approaches for controlling cervical cancer incidence have paved in parallel routes for a while; secondary prevention implementing cytology and HPV-related biomarkers for
Volume 7 Issue 4 - 2017

\author{
Georgios Androutsopoulos, Georgios \\ Michail, Georgios Decavalas \\ Department of Obstetrics and Gynecology, University of Patras, \\ Greece
}

Correspondence: Georgios Androutsopoulos MD, PhD, Assistant Professor, Department of Obstetrics and Gynecology, University of Patras, Medical School, Rion 26504, Greece, Tel +306974088092, Email androutsopoukos@upatras.gr

Received: February 16, 2017| Published: March 03, 2017

identifying precancer lesions, with subsequent resort to colposcopy; and HPV vaccination-virtually the incarnation of primary prevention. Even in the western world however, cervical screening is commonly opportunistic; or might be organized inconsistently across and within countries and regions.7 In an excellent review addressing the flaws of secondary cervical prevention, the authors point at the differences between organized and opportunistic screening; in the former the basic principle is that the more women screened (as differentiated from the more tests done), the less morbidity and mortality from cervical cancer. ${ }^{7}$ In contrast, opportunistic screening is often characterized by the over-screening of a minority of (some privileged) women, while those women most at risk tend to be screened rarely or not at all. ${ }^{7}$ Thus, across all levels of screening coverage, increases in test sensitivity provide greater reductions in the lifetime risk of cancer, but the magnitude of cancer risk reduction depends on the level of coverage. ${ }^{8}$ Inevitably, opportunistic and organized programs intersect in a variety of ways and the authors acknowledge that in all jurisdictions with organized screening programs, at least some (and often a great deal of) opportunistic screening is also undertaken, and data from that opportunistic screening are often not captured. ${ }^{7,8}$

None of the theoretical advantages of HPV vaccination strategies which were initially proposed at the time of the vaccine launch has been effectively annulled; on the contrary, most have been widely corroborated. In terms of secondary prevention efficacy, several metaanalyses have corroborated the results of Soutter et al., who pointed that the relative risk for cervical cancer among women treated for any grade of precancer was several times that of the background risk for the next decade or more following treatment, justifying the need for sustained vigilance. ${ }^{9}$ This elevated risk might be higher in women treated for high-grade lesions or who test hr-HPV positive. ${ }^{10}$

Likewise, the consequences of various surgical treatment modalities of screen-detected cervical precancer in neonatal prematurity and perinatal morbidity have been highlighted in seminal research that was published almost concurrently with the launch of the 
quadrivalent VLP HPV vaccine and paved the way to a new subchapter in cervical pathology. ${ }^{11,12}$ Irrespective of whether those concerns have occasionally caused excisional treatments of insufficient depth, this research has drawn attention among physicians to avoid re-cones, tophats and other undue overtreatment.

Cervical adenocarcinoma is globally on the rise, and one possible explanation could be the prolonged use of hormonal contraception; not surprisingly intrauterine contraceptive devices might have a negative correlation with the development of adenocarcinoma. ${ }^{13}$ The contribution of HPVs in glandular lesions has been a topic where much insight has been gained in the past decade; we now know that almost all adenocarcinomas arising from the cervix are hr-HPV-related. ${ }^{14}$ Despite the enhanced detection rates of glandular precancer with the implementation of LBC, glandular lesions arising in the cervix are still easily missed, either due to localization high in the canal, or due to their multifocal nature; yet another era where primary prevention clearly trumps.

The psychological consequences of the treatments for cervical precancer represent a large and hugely underestimated area of interest. This wealth of negative feelings and sentiments has been the subject of exhaustive research, and quite often the emotional trauma is more important than the physical one. But then, it's not only the treatment that provokes anxiety and negative feelings, it's also the HPV infection itself, that induces stigmatization.

Real world declines in the prevalence of HPV-related outcomes are also established. Decreases in warts prevalence in nations which implemented centrally organized vaccination programs represent an early benefit of such vaccination programs. ${ }^{15}$ Decreases in the rates of detected high grade precancerous abnormalities in young women have been already documented. ${ }^{16}$ Besides, evidence of cross-type protection, primarily for HPV 45 and, to a lesser extent, for HPVs 31 and 33 has been also documented. ${ }^{17}$

The causative relation of HPV infection with the development of neoplasms in other sites, namely vulvar, penile, oropharyngeal and anal cancer, that has been only lately corroborated, was not yet established at the time of launch of the first generation VLP vaccines. ${ }^{18}$ These relations translated to welcome bonus gains in public health prevention strategies, which redefine the logistics and justify the costs imposed by the vaccination programs. Of course, this additional morbidity burden was irrelevant and cannot be dealt by the secondary cervical prevention.

Regarding the effect of herd immunity, this is more pronounced in populations where high HPV-vaccination coverage has been achieved; in these populations, substantial reductions in the rates of infection and cervical neoplastic disease have been observed not only among those women who received the vaccine, but also among the non-vaccinated females and males aged $<25$ years. This is the case for pioneers in vaccination, like Australia. ${ }^{19}$ At a certain herd immunity level, tailoring screening to vaccination status may no longer be justified, as the risk level becomes cost-ineffective for unvaccinated women. This herd immunity effect also pertains solely to primary prevention. ${ }^{20}$

Regarding the initial concerns on vaccine safety, after the administration of tenths of millions doses of all three brands of HPV vaccines, VLP vaccine safety profile has been established and does not represent an issue. ${ }^{21}$ The cost-effectiveness of a combined primary and secondary cervical prevention programs are critically dependent on the age of vaccination and initialization of screening, screening frequency, and the intensity of conducting further tests for mild cytologic abnormalities. ${ }^{22}$ Cost-effectiveness models will help determine the optimal combination of HPV vaccination and screening in public health programs, and balance the effects of such approaches in different populations. ${ }^{19}$ With the increasing demand under pressing health logistics for less screening in well-HPV-vaccinated cohorts (ie, those vaccinated primarily before becoming sexually active), strategies that combine HPV vaccination with simplified protocols of HPV screening and cytology triage, at least for women aged $>30$, will be of interest in many settings in the foreseeable future. ${ }^{19,23,24}$ Besides, as herd immunity levels increase, the core design of screening schedules (e.g., screening intervals or screening start age) evolves, highlighting the importance of jointly optimizing both primary and secondary prevention strategies. ${ }^{25,26}$

Finally as El-Zein and colleagues argue, decision-making nowadays on cancer screening incorporates not only the extent of benefit in mortality reduction but also the potential harms from screening. ${ }^{27}$ Policymakers need to assess related costs, utilities, infrastructures, political risks of inaction and patient choices. ${ }^{27}$ Health benefits, lifetime costs and screening harms in terms of referrals and overtreatments need to be balanced.

\section{Acknowledgments}

None.

\section{Conflicts of interest}

Authors declare there are no conflicts of interest.

\section{References}

1. Collins Y, Einstein M, Gostout B, et al. Cervical cancer prevention in the era of prophylactic vaccines: a preview for gynecologic oncologists. Gynecol Oncol. 2016;102(3):552-562.

2. Khalid S, Carcopino X, Michail G, et al. Compliance with follow up cytology after discharge from the colposcopy clinic. Ir Med J. 2011;104(6):167-170.

3. Woo Y, Omar S. Human papillomavirus vaccination in the resourced and resource-constrained world. Best Pract Res Clin Obstet Gynaecol. 2011;25(5):597-603

4. Regan D, Hocking J. Greatest effect of HPV vaccination from schoolbased programmes. Lancet Infect Dis. 2015;15(5):497-498.

5. Dijkstra M, Snijders P, Arbyn M, et al. Cervical cancer screening: on the way to a shift from cytology to full molecular screening. Ann Oncol. 2014;25(5):927-935.

6. Cubie H, Cuschieri K. Understanding HPV tests and their appropriate applications. Cytopathology. 2013;24(5):289-308.

7. Williams J, Carter S, Rychetnik L. 'Organised' cervical screening 45 years on: How consistent are organised screening practices? Eur $J$ Cancer. 2014;50(17):3029-3038.

8. Goldie S, Kim J, Myers E. Chapter 19: Cost-effectiveness of cervical cancer screening. Vaccine. 2006;24(Suppl 3):S3164-S370.

9. Soutter W, Sasieni P, Panoskaltsis T. Long-term risk of invasive cervical cancer after treatment of squamous cervical intraepithelial neoplasia. Int $J$ Cancer. 2006;118(8):2048-2055.

10. Ghaem-Maghami S, De-Silva D, Tipples M, et al. Determinants of success in treating cervical intraepithelial neoplasia. BJOG. 2011;118(6):679-684. 
11. Kyrgiou M, Koliopoulos G, Martin-Hirsch P, et al. Obstetric outcomes after conservative treatment for intraepithelial or early invasive cervical lesions: systematic review and meta-analysis. Lancet. 2006;367(9509):489-498.

12. Arbyn M, Kyrgiou M, Simoens C, et al. Perinatal mortality and other severe adverse pregnancy outcomes associated with treatment of cervical intraepithelial neoplasia: meta-analysis. BMJ. 2008;337: a1284.

13. Castellsague X, Diaz M, de Sanjose S, et al. Worldwide human papillomavirus etiology of cervical adenocarcinoma and its cofactors: implications for screening and prevention. J Natl Cancer Inst. 2006;98(5):303-315.

14. Holl K, Nowakowski A, Powell N, et al. Human papillomavirus prevalence and type-distribution in cervical glandular neoplasias: Results from a European multinational epidemiological study. Int $J$ Cancer. 2015;137(12):2858-2868.

15. Mariani L, Vici P, Suligoi B, et al. Early direct and indirect impact of quadrivalent HPV (4HPV) vaccine on genital warts: a systematic review. Adv Ther. 2015;32(1):10-30.

16. Brotherton J, Fridman M, May C, et al. Early effect of the HPV vaccination programme on cervical abnormalities in Victoria, Australia: an ecological study. Lancet. 2011;377(9783):2085-2092.

17. Malagon T, Drolet M, Boily M, et al. Cross-protective efficacy of two human papillomavirus vaccines: a systematic review and meta-analysis. Lancet Infect Dis. 2012;12(10):781-789.

18. Gillison M. Human papillomavirus-related diseases: oropharynx cancers and potential implications for adolescent HPV vaccination. $J$ Adolesc Health. 2008;43(4 Supp1):S52-S60.
19. Bosch F, Robles C, Diaz M, et al. HPV-FASTER: broadening the scope for prevention of HPV-related cancer. Nat Rev Clin Oncol. 2016;13(2):119-132.

20. Naber S, Matthijsse S, Rozemeijer K, et al. Cervical Cancer Screening in Partly HPV Vaccinated Cohorts - A Cost-Effectiveness Analysis. PLoS One. 2016;11(1):e0145548.

21. Gee J, Weinbaum C, Sukumaran L, et al. Quadrivalent HPV vaccine safety review and safety monitoring plans for nine-valent HPV vaccine in the United States. Hum Vaccin Immunother. 2016;12(6):1406-1417.

22. Garnett G, Kim J, French K, et al. Chapter 21: Modelling the impact of HPV vaccines on cervical cancer and screening programmes. Vaccine. 2006;24(Suppl 3):S3178-S186.

23. Castle P. When Less is More. J Natl Cancer Inst. 2007;109(2).

24. Kim J, Burger E, Sy S, et al. Optimal Cervical Cancer Screening in Women Vaccinated Against Human Papillomavirus. J Natl Cancer Inst. 2016;109(2).

25. Michail G, Androutsopoulos G, Decavalas G. Cervical cancer surveillance - Considerations in the vaccination era. Int J Community Fam Med. 2016;1(2):117.

26. Sander B, Wong W, Yeung M, et al. The cost-utility of integrated cervical cancer prevention strategies in the Ontario setting - Can we do better? Vaccine. 2016;34(16):1936-1944.

27. El-Zein M, Richardson L, Franco E . Cervical cancer screening of HPV vaccinated populations: Cytology, molecular testing, both or none. $J$ Clin Virol. 2016;76(Suppl 1):S62-S68. 